THE INTERNATIONAL

REVIEW OF RESEARCH IN

OPEN AND DISTANCE LEARNING

\title{
Learner Analysis Framework for Globalized E-Learning: A Case Study
}

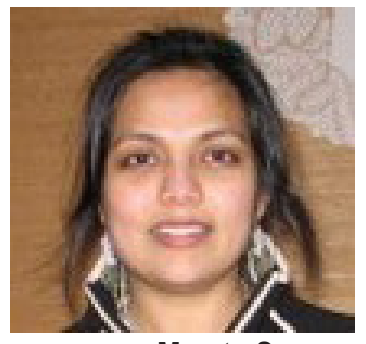

Mamta Saxena

Southern New Hampshire University, USA

\section{Abstract}

The shift to technology-mediated modes of instructional delivery and increased global connectivity has led to a rise in globalized e-learning programs. Educational institutions face multiple challenges as they seek to design effective, engaging, and culturally competent instruction for an increasingly diverse learner population. The purpose of this study was to explore strategies for expanding learner analysis within the instructional design process to better address cultural influences on learning. A case study approach leveraged the experience of practicing instructional designers to build a framework for culturally competent learner analysis.

The study discussed the related challenges and recommended strategies to improve the effectiveness of cross-cultural learner analysis. Based on the findings, a framework for conducting cross-cultural learner analysis to guide the cultural analysis of diverse learners was proposed. The study identified the most critical factors in improving cross-cultural learner analysis as the judicious use of existing research on cross-cultural theories and joint deliberation on the part of all the participants from the management to the learners. Several strategies for guiding and improving the cultural inquiry process were summarized. Barriers and solutions for the requirements are also discussed.

Keywords: Distance learning; learner analysis; cultural analysis; globalized e-learning; learner analysis frameworks; instructional design 


\section{Introduction to the Study}

Learner analysis is a critical aspect of instructional design and is characterized as an iterative process that informs vital instructional design decisions from front-end analysis to evaluation (Edmundson, 2007; Morrison, Ross, \& Kemp, 2004; Rogers, Graham, \& Mayes, 2007). At this time, there is a limited body of research addressing the role of cultural background within the process of learner analysis, and unfortunately traditional instructional design processes are inadequate in informing the design of cross-cultural instruction (Henderson, 1996; McLoughlin, 1999, Rogers et al., 2007). Effective design for global distance learning is in need of further research (Gunawardena, Wilson, \& Nolla, 2003; Mason, 2003). The natural path to achieving this goal is through a re-examination of existing models and strategies of learner analysis via the lived experience of instructional designers.

The purpose of this study was to investigate the variability in existing frameworks of cultural analysis within the instructional design practice as described and practiced by experienced instructional designers from differing cultural backgrounds working in a global distance learning setting. The ultimate goal of the study was to propose a framework for improving the effectiveness of culturally competent learner analysis. This goal was realized through a qualitative study that was guided by the following research questions:

1. To what extent do instructional designers conduct cultural inquiry during their analysis of learner characteristics?

2. In what ways are instructional designers currently considering and addressing cultural influences in their design process?

3. How do instructional designers characterize the role of learner analysis in creating culturally appropriate distance learning content?

4. How do designers define the elements of cultural analysis critical for culturally competent design?

\section{Brief Review of the Literature}

From an educational perspective, culture plays a crucial role in pedagogical values, learning styles, and cognitive processing (Smith \& Ayers, 2006). When teaching and learning take place within a single cultural context, the unity of experience and cultural background is generally unobtrusive. When instruction begins to reach individuals of multiple cultural backgrounds, however, there is a risk of creating barriers based on the inability to address or mediate differences in educational values and cultural beliefs embedded in the content and multimedia. These differences can include perceptions about the roles and responsibilities of instructors and learners, prevailing styles of teaching and learning, and nuances of language and semantics (Chen, Mashadi, Ang, \& Harkrider, 1999; Henderson, 1996; McLoughlin, 1999; Rogers et al., 2007; Smith \& Ayers, 2006). 
While such challenges would be present in any classroom that combines individuals from varying cultural backgrounds, the additional challenges presented by distance learning present an extra layer. Chen, Mashadi, Ang, and Harkrider (1999) claimed that even if one overcomes the instructional design-related issues of distance learning, the value of learning mainly depends on the learner's experiences of cultures and technology. Instructional designers must factor in these differences when designing instruction for a diverse learner population to reduce the impact of cultural barriers on effective learning. In addition, instructional designers have to be cognizant of their own predispositions based on worldviews.

Based on some of the expert viewpoints in the field of instructional design (Bentley, Tinney, \& Chia, 2005; Henderson, 1996; Henderson, 2007; McLoughlin, 1999; Rogers et al., 2007), a culturally competent design can be defined as one that strives to effectively mediate culture in order to fulfill the needs and expectations of the majority of learners. Such a design must be built upon an eclectic pedagogic paradigm and shared epistemological systems to create a distance learning environment that (a) is sensitive to the cultural differences of learners and factors in the cultural differentials of language, technology, cognitive processing, cultural context, and motivational factors; (b) makes the educational values of the participants (both learners and designers) explicit upfront; and (c) offers flexibility in regard to the pedagogies, resources, and delivery strategies and provides additional support based on learner needs.

Such a design aims to create a user-centered learning environment and is based on welldefined learner needs and expectations. One may argue against the possibility of a true culturally competent design considering the myriad of cultures and subcultures that exist, the protean nature of those cultures, and the inherent challenges of technology-mediated instruction. Nevertheless, diligent market research, constantly emerging and evolving technologies, and shared accountability on the part of the designers, the learners, and the research community can assist in sorting, validating, and creating a repository of intercultural dimensions to create a framework for culturally competent design that can reach the majority of learners. The first step to achieving this goal is designing a framework for cultural competence within the context of distance learning, and this necessarily begins with expanding the current approach to learner analysis (Rogers et al., 2007).

\section{Research Method}

A multiple case study approach explored designers' views on cultural inquiry of distance learners and their perceptions about how specifically the data can be gathered and used for making design and delivery decisions for cross-cultural instruction. An in-depth analysis of multiple cases revealed the current practices among instructional designers in the pursuit of culturally competent design and their perceptions about current learner analysis strategies and about the learner information that is most valuable in incorporating cultural factors into design. 


\section{Participant Characteristics}

The study involved eight instructional designers from varied educational and business backgrounds. Six respondents were from the United States, one was from the United Kingdom, and one was from India. The designers had wide-ranging experiences of cross-cultural design working for a variety of corporate training environments, such as healthcare, financial services, insurance, and corporate universities. Participants ranged from independent contractors and self-employed e-learning consultants to corporate designers of large international companies.

Cross-cultural design experience also varied in that some participants had extensive or exclusive experience designing and delivering training for learners from a multitude of nations and cultures, while the experience of others was limited to designing for learners from different cultures within a given country. All participants had five or more years of experience in design, including distance learning, and in-depth knowledge and familiarity with the needs assessment process.

\section{Data Collection}

Sixty-minute telephone interviews were conducted with each subject, and the interviews were recorded for transcription. A semi-structured interview format was used, based on the research questions that provided the focus for this study. Collection took place over a period of two months in 2009.

\section{Data Analysis}

Patterns within the responses made it possible to identify the following: prevailing themes that provided insight into current practices of cultural analysis, needs specified by instructional designers for more effective incorporation of culturally relevant components in the analysis process, and factors of influence with regard to culturally competent design. A multiple case study approach offered an opportunity to gather insights from within each case and across cases for replication and contrasting findings and is considered more compelling and robust than a single case study approach (Yin, 2003).

Data analysis and exploration of themes were based upon the textual and conceptual functionality of coding in Atlas.ti and the qualitative analysis methods for cross-case analysis proposed by Miles and Huberman (1994). The analysis included the following processes: (a) coding and within-case analysis; (b) cross-case exploration using partially ordered display tables; (c) cross-case explanation and prediction via conceptually ordered content-an-

alytic summary tables, fragment causal networks, and narratives; and (d) meta-analysis of fragment causal networks. Trustworthiness and credibility of the findings were established by using member checking, thick descriptions, and peer debriefing.

A provisional start list of codes was developed, drawn from the conceptual framework, research questions, literature review, and key variables brought to the study. The a priori codes were based on Bogdan and Biklen's (1992) scheme of defining codes in the following ways: (a) setting or context, (b) definition or situation, (c) perspectives, (d) ways of thinking 
about people and objects, (e) process, (f) activities, (g) events, (h) strategies, (i) relationships and social structure, and (j) methods. These codes were collapsed into first level codes and then into family codes. These code families served as constructs identified under the themes as follows: (a) definition, (b) process, (c) cultural accommodations, (d) perception, (e) challenges, and (f) strategies. Constructs provide order to the descriptive data as they are concepts that are derived from observed phenomena and can be used to explain those phenomena.

\section{Findings Related to Cultural Analysis}

Data analysis afforded insights into the current challenges associated with conducting cross-cultural learner analysis and revealed various strategies that designers are either currently using or believe would be effective. The outcomes of the data analysis in the metacausal network identified the following needs:

1. define the role of cultural analysis,

2. reformulate approach and focus for cultural analysis,

3. redefine the role of the participants and the sponsors, and

4. reassess training and information needs.

\section{Define the Role of Cultural Analysis}

Each participant offered a different definition of culture with some commonalities. While some associated culture with the organizational culture that learners come from, others linked culture with nationality or ethnicity and language; still others interpreted cultural differences as defined by personal learning styles. The feedback from the participants helped create an extensive list of cultural variables critical for distance learning design, from language and technical adaptability to religion, cultural taboos, and etiquette, but also revealed the absence of any universally applicable definition of culture. The participants' perceptions of cross-cultural design revealed a strong sense of cultural inclusivity among designers and the desire to learn more about learners' cultural needs, but because each defined culture differently there was no common approach.

There appeared to be a lack of clarity on (a) how to precisely define culture and diversity in the teaching-learning context; (b) how to delineate the cultural variables that are critical to include during learner analysis; (c) how to determine the expectations of the participants, from the sponsors and the designers to the learners and the research community, as to how much of the cultural analysis is necessary to be able to create a culturally competent design; and (d) how to approach the cultural analysis process with no defined framework or model.

According to some of the participants, the awareness of the need to invest time and money in cultural analysis is absent among the key stakeholders: the sponsors. Even if the design- 
ers and the management acknowledge the need, personal bias or ignorance may lead to a lack of acceptance or recognition of the cultural issues, and this might also contribute to resistance by sponsors and designers. For example, Case 5 explained,

customers hire me to discover why training is not working in other cultures and when I tell them I do not know why they do not quite believe it and I am not sure how to get them past that hurdle. It must be an American thing that I must be doing everything right. (\$49)

The findings align with the conclusions drawn by many studies that divulged the natural embodiment of a pro-Western bias in distance learning (Chen et al., 1999; Henderson, 1996; McLoughlin, 1999a; Rogers et al., 2007; Smith \& Ayers, 2006).

The findings from the cases affirm that there is often a lack of knowledge and awareness among the designer community about how to approach the process of cultural analysis. Therefore, there is a critical need for the instructional design community to clearly define the role of cultural analysis in the needs assessment of diverse learners for cross-cultural design, and this call to action is echoed by other researchers such as Henderson (1996), McLoughlin (1999a), and Rogers et al. (2007).

\section{Reformulate the Approach and Focus for Cultural Analysis}

Analysis of the construct related to the perceptions of designers about cross-cultural design and cross-cultural learner analysis revealed that the designers agreed with the need to address cultural issues. However, even though the designers had a strong desire to learn more about the cultural needs of learners, their current practice does not have a clearly defined approach and focus for such an analysis during the needs assessment process. Whether it be due to the time, budget, and resource constraints or because of bias and ignorance, the sponsors and the management are unable to precisely understand and support cultural analysis as delineated by the designers.

\section{Redefine the Role of Participants}

The designers reflected an understanding of the urgent need to gather critical cultural data on learners but noted that they were bound by organizational expectations and policies, personal bias, and a lack of knowledge on (a) how to approach the process, (b) what exactly to look for in cultural analysis, and (c) how to translate the information in cross-cultural design.

As well, the absence of direct input from the learners is of significant concern. The majority of the participants acknowledged that learner information is provided by the sponsors or clients or gathered via other sources with no direct learner input. Some of the designers, however, expressed a strong desire to include the learner in the process, either before or after delivery, to understand the learning gaps created due to cultural differences. Designer 5 asserted that even an otherwise thorough cultural analysis process is incomplete without learners' input. Hence, there is a need to redefine the role of learners as active participants 
in the cultural analysis process, whether it is to gather new information or to validate the assumptions based on research information.

\section{Reassess Training and Information Needs}

Some of the participants expressed the need for a database or knowledge base on cultural variables or cultural profiles of learners, based on nationality or other criteria that impact learning, that could serve as a reference. The designers had a strong desire to learn about cultural needs, but they expressed the need for access to research-based information in an organized form, such as in a knowledge base related to cross-cultural design principles or cultural variables. The research community and professional organizations can help by disseminating research information efficiently and making it easily available for designers and learners.

Other participants articulated the idea of learning through training workshops, if not through extensive training, in cross-cultural design. Currently, there are no mandatory training or competency requirements for designers to be skilled in cross-cultural design or cultural analysis. Because there are no standards, the participants were not only ambivalent about what it means to be culturally competent but also intimidated by the idea of trying to address a myriad of cultures. Therefore, a readily available data source would assist in addressing some of the aforementioned concerns.

Also, the designers expressed the need for a framework or model for cross-cultural learner analysis with clearly outlined steps in the process for designers. Some of the participants pointed out the difficulty in having access to cultural expertise. Redefined training and information resources might bring a level of cultural expertise to the designers that would augment their existing repertoire of cross-cultural design skills.

The needs and requirements for competent cross-cultural design based on the data are illustrated in Figure 1. It is important to note both the individual elements within the framework and the relationships among the various parts. The intricacies of the framework reflect the intrinsically complex nature of cultural analysis and highlight the varied knowledge and skills designers require to make the practice a core element of their design approach. 


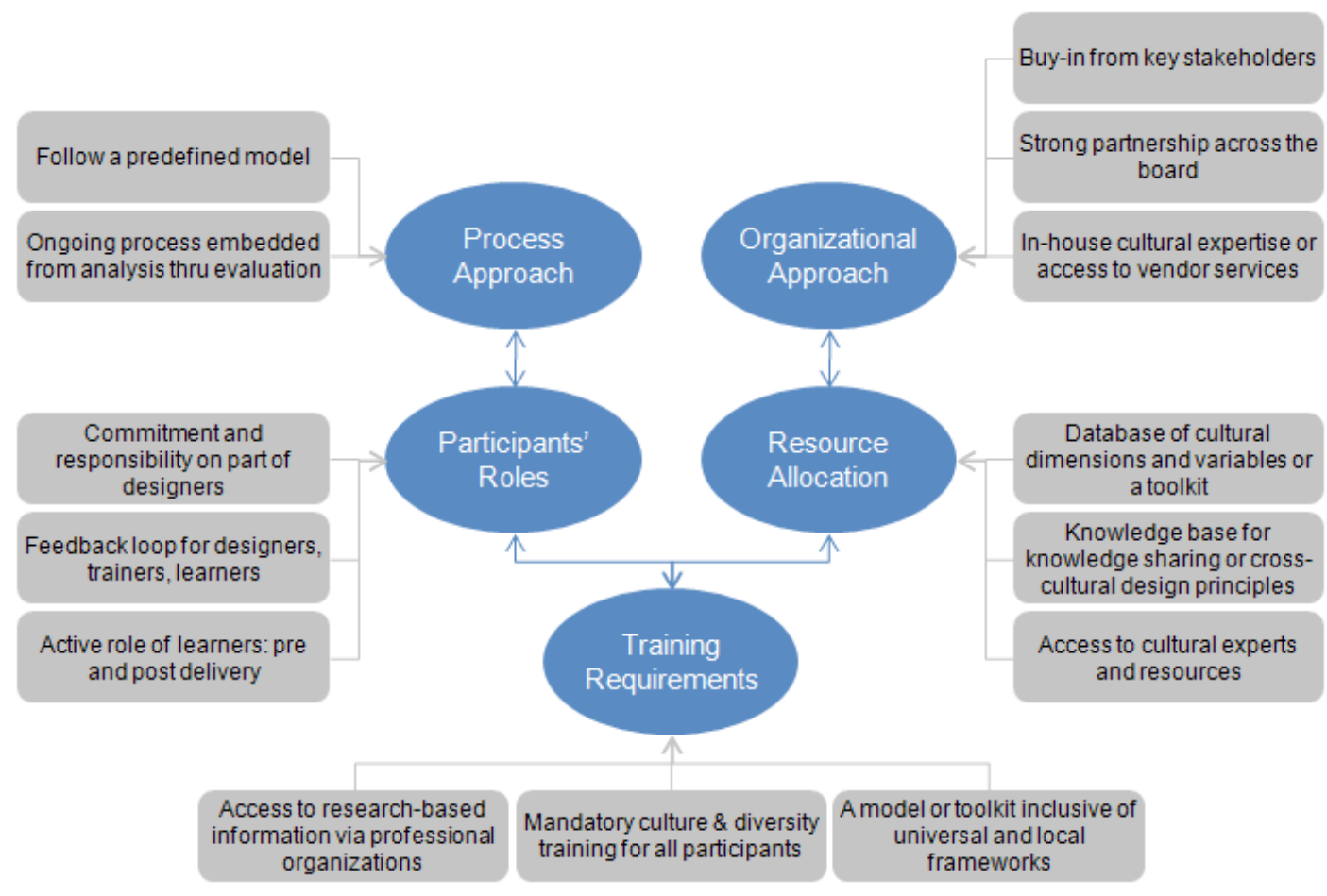

Figure 1. Needs and requirements for cross-cultural learner analysis.

\section{Barriers, Solutions, and the Proposed Framework}

The barriers and solutions identified by the designers fell into three categories: (a) organizational, (b) procedural, and (c) personal.

\section{Organizational Barriers and Solutions}

Organizational challenges faced by designers include difficulty in getting buy-in from key stakeholders, in obtaining ongoing support, and in accessing necessary resources. The proposed solutions for the aforementioned barriers include educating management, making a strong business case for cultural analysis, and establishing a strong partnership across the board. Designer 5 commented on the challenges, stating,

I would say it is still probably mostly organizational because while I will often work with someone in the organization who understands the value in what we are doing and that it will save the company time or money or save a relationship, it is often not the highest level in the company: so they often do not get buy-in. ( $\left(\|_{100)}\right.$

One way of addressing this concern is through mandatory workshops or seminars for leaders and management about the significance of cultural analysis in providing effective, targeted training to global learners. 
Another solution is to help business leaders and managers become more culturally intelligent themselves. Thomas and Inkson (2009) defined cultural intelligence (CQ) as

being skilled and flexible about understanding a culture, learning more about it from your ongoing interactions with it, and gradually reshaping your thinking to be more sympathetic to the culture and developing your behavior to be more skilled and appropriate with others from the culture (p. 17).

They included three variables in CQ: knowledge, mindfulness, and skills about culture and cross-cultural competence in varying intercultural scenarios. It is anticipated that as personal cultural intelligence rises, so too does a positive disposition toward cultural analysis.

Additionally, it is important to make a business case for cultural analysis for the sponsors and clients. Designer 5 suggested presenting cultural analysis as a critical part of market analysis, while another designer recommended that it be embedded in the needs assessment process.

When you are designing learning and you want it to be successful in another country or culture WHY would you NOT explore this aspect and not include as otherwise you are setting yourself to defeat for market loss, money loss. I say it is just part of market analysis. In that respect sometimes we get a few people who understand right from the beginning and they let us make those changes but that is really rare. $(\Phi 164)$

If key stakeholders understand and appreciate the need for cultural analysis, organizations will have a clearly defined role for cultural analysis and it will become an essential part of the needs assessment. Designers will then have access to the resources they need to conduct an effective cultural analysis process that gathers critical cultural data on learners.

\section{Procedural Barriers and Solutions}

The procedural barriers in cross-cultural learner analysis include an unclear approach and focus, time and cost limitations, resource constraints, and feasibility issues.

\section{Approach and focus.}

One of the important steps in addressing the time constraints is to explain to the participants, especially the sponsors, the need to approach cultural analysis as an ongoing process that is embedded in the instructional design process from needs assessment through evaluation and redesign. Because globalization is a relatively new phenomenon and culture is a multifaceted issue, questions dealing with cultural analysis will not have easy answers. Designer 5 noted, 
Probably one of the barriers is inability or unwillingness to accept that this is a research process. Americans usually want quick answers: how do I fix it. They just want to go to a book and real quick say here look this is the answer and move on. ( $(181)$

However, with the right approach and focus, the process of cultural analysis can be defined for the targeted audience and executed in a time-efficient manner. For this, it is critical to craft a framework and a model that outlines the essential steps of the formal process and delineates the scope so the designers can take the time upfront to learn about the cultural makeup of the audience based on that predefined model. The model can be built upon the existing learner analysis variables, such as gender, age, ethnicity, and language, but it also needs to incorporate existing research to add other cultural variables that have significant impact on the content, delivery method, pedagogy, and technological aspects of the course.

\section{Time, cost, and resource constraints.}

The issue of resource and time constraints can be tackled by making information on cultural profiles and issues easily accessible for designers to bring cultural expertise to the design and analysis process. Establishing a database created by the support of a research community and building professional organizational networks dedicated to cross-cultural design and analysis is the first step. The goal of such a database would be to provide a repository of varied philosophical, pedagogical, cultural, and epistemological underpinnings of diverse learners based on predefined categories. Such a database would serve as a knowledge base for practitioners and researchers worldwide.

Although the potential benefit of such a repository is significant, the need for cultural analysis does not end with the initial design. Cultural dimensions based on national cultures and other models will help identify some characteristics of the cultural profile of global learners, but focus on intercultural communications and interactions in the online course room itself is essential. Hence, what becomes critical in shaping the cyber-identity of the learner is the communication or interactions in which they are involved, so it is important that not only the designers but also the facilitator and the learners be aware of the cultural profiles of learners to make sense of the diversity in cyberspace.

\section{Feasibility issues.}

One of the arguments against establishing a cultural knowledge base is the feasibility of the practice in terms of effort, time, and money needed for gathering information about different cultural theories and profiles of learners across the globe. Designer 3 conducted a full-scale learner analysis to create a diversity training program for a company's leaders spread across the globe. When questioned about the applicability of the findings from that study to other training programs, the designer answered,

Yes it is doable, it could be replicated at a lower cost, 
and this was costly due to false starts. If we have to do it again, both with the hindsight or my experience we would have avoided some of the re-dos and I would lend that knowledge to other people how they could avoid those redos. $(\boldsymbol{\Phi} 170)$

When asked about the reuse of the knowledge discovered in one project for another, the designer commented, "the research that went into creating this and expertise used to create the content can be used to create other training" ( $\mid 178)$. Thus, with solicitous market research, strong institutional support (Alexander, 2002; Bates \& Gpe, 1997), and a shared vision by the online distance education community, the goal is attainable.

\section{Personal Barriers and Solutions}

One of the major issues related to personal challenges is the absence of well-defined training or knowledge-sharing programs for the participants, especially the designers. Data analysis revealed that most of the participants were not aware of existing research and models that can address cultural issues to some degree and offer guidelines for cross-cultural design. Such existing models include the multiple cultures model (MCM) proposed by Henderson (1996), the cultural adaptation process (CAP) model by Edmundson (2007), and the universal design for culturally diverse online learning framework proposed by Eberie and Childress (2007).

Cultural issues must be part of training and knowledge for not only the designers but also for management and the learners. Awareness and knowledge of differing cultures will have a direct impact on personal biases of the participants and help build cultural intelligence (Thomas \& Inkson, 2009). Nonetheless, personal bias is something designers need to be aware of so they can acknowledge it and then try to address it if they are truly committed to creating culturally competent design. Designer 5 pointed out that "unless designers are truly committed to understanding the differences and accepting the fact that it is making a

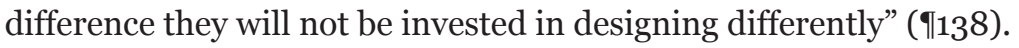

The discussion of identified barriers and their potential solutions resulted in the emergence of key themes that form recommendations for enhancing cross-cultural competence for instructional design in distance learning. These include the following:

1. establishing well-defined cross-cultural competencies for all the participants,

2. developing and implementing mandatory cross-cultural education and training for all the participants,

3. implementing a tested model or toolkit for cross-cultural learner analysis,

4. building an established knowledge base for cross-cultural design and analysis, and

5. developing and distributing a database of cultural dimensions or profiles. 
These provisions, in concert with established best practices for instructional design, helped craft the proposed framework (see Figure 2) for improving the effectiveness of cross-cultural learner analysis to facilitate the design of culturally competent courseware.

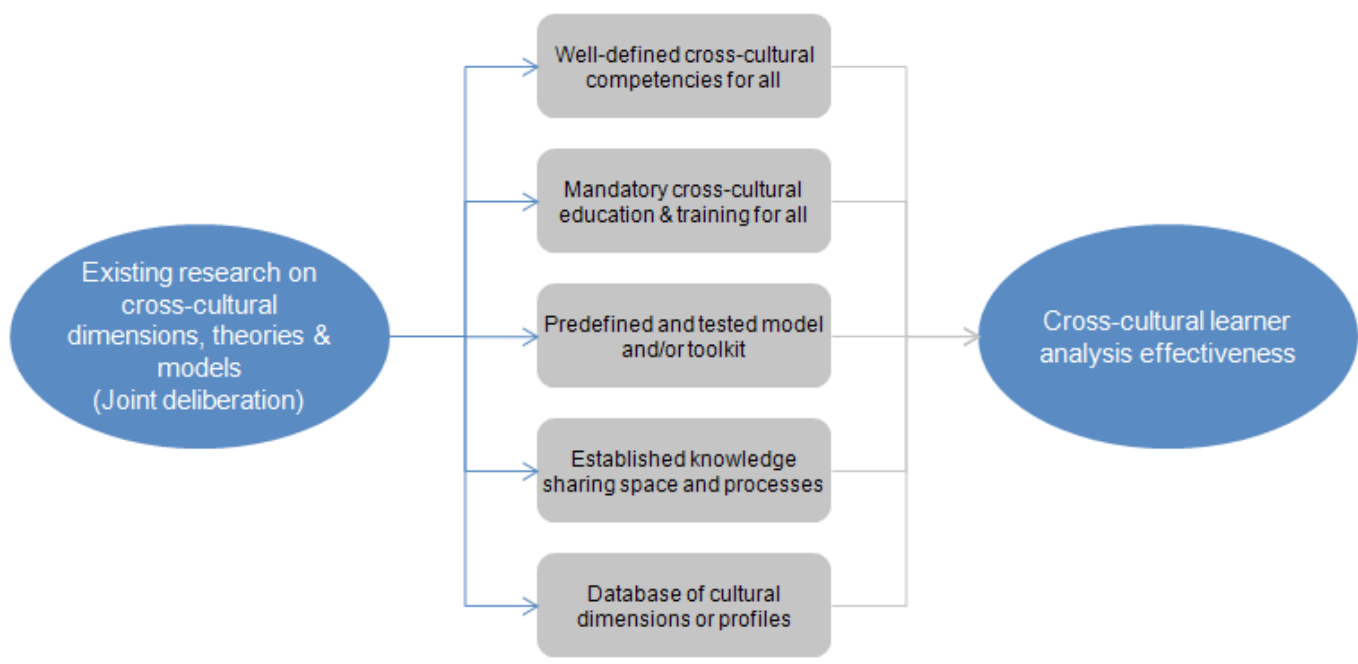

Figure 2. Cross-cultural learner analysis framework.

\section{Limitations}

All of the eight designers interviewed for the study were working for corporate organizations, and most were from the United States. Hence, generalizations of the findings to other types of organizations will be limited, even though the participants came from a wide range of design backgrounds, from large global corporate companies to independent contractors and from designers working solely online to ones designing for a blended learning environment. The small participant pool makes it unlikely that all views of instructional design professionals have been captured.

Moreover, since the findings were based on data collected from participants' personal perceptions of cross-cultural analysis and design, the results may be biased. It is also important to consider the proposed framework in regard to its applicability and feasibility, depending on an organization's customer base, training philosophy, distance learning approach, and leadership style. The elements in the framework do not present an exhaustive list but offer an initial set of variables that need attention to begin improving the effectiveness of crosscultural learner analysis that ultimately supports culturally competent courseware.

\section{Conclusion and Directions for Future Research}

The findings of this cross-case analysis revealed many of the often unspoken barriers to cross-cultural design. The acknowledgement and acceptance of the need for cross-cultural learner analysis is one of the most significant steps toward improving cross-cultural analy- 
sis, and this study revealed the strong sense of inclusivity among the designers, which was limited largely by organizational and procedural barriers that, although present, are not insurmountable.

The findings produced an inclusive list of approaches to address the barriers related to cross-cultural learner analysis and illustrated the gaps in practice and existing research to reveal that existing resources could be organized in an accessible, systematic fashion to advance and promote cultural considerations within learner analysis. The participants in this study were universal in their agreement that there is an urgent need for joint deliberation on the part of the instructional design community, the research community, and the learners to craft a framework for effective cross-cultural learner analysis. This study was intended to initiate this dialog, and as solutions are proposed, new questions must be considered in subsequent discussions:

1. What are the potential barriers to each one of the proposed elements of the framework as perceived by leaders, management, designers, researchers, and learners? How can they be overcome?

2. What are the cross-cultural learner analysis processes followed by corporate companies in other nations?

3. How can designers make a strong business case to prove that investment in cross-cultural learner analysis will be financially worthwhile in the long run?

4. How can cultural profiles based on nationality or cognitive processing be expanded and how can learning be measured in relation to the key cultural variables?

5. How are cultural identities based on nationality altered in cyberspace to become a third identity? Does the alteration hinder or facilitate learning?

This study provides a foundation for future research that addresses all of these questions. Perhaps most needed is additional research that will validate and expand the proposed framework.

The global distance learning community must work together to create a process that addresses cross-cultural analysis and design for global learners. The purpose in presenting the model is to persuade organizations and instructional design professionals to acknowledge the need for cultural considerations and to seek and incorporate such knowledge within their instructional design practice. The global reality of distance education means that instructional design of any form and in any part of the world must consider the cultural diversity inherent to it. This study is an invitation to initiate discussion on how to address this reality. 


\section{References}

Alexander, S. (2002). Designing learning activities for an international online student body: What have we learned? Journal of Studies in International Education, 6(2), 188-200.

Bates, A. W., \& Gpe, J. Escamilla de los Santos. (1997). Crossing boundaries: Making global distance education a reality. Distance Education, 12(1/2), 49-66. Retrieved from http://www.tonybates.ca/papers/crossingboundaries.html

Bentley, J. P. H., Tinney, M. V., \& Chia, B. H. (2005). Intercultural Internet-based learning: Know your audience and what it values. Education Technology Research and Development, 53(2), 117-127.

Bogdan, R., \& Biklen, S. K. (1992). Qualitative research for education: An introduction to theory and methods (2nd ed.). Boston: Allyn \& Bacon.

Chen, A. Y., Mashadi, A., Ang, D., \& Harkrider, N. (1999). Cultural issues in the design of technology-enhanced learning systems. British Journal of Educational Technology, 3o(3), 217-230.

Eberie, J. H., \& Childress, M. D. (2007). Universal design for culturally-diverse online learning. In A. Edmundson, Globalized e-learning cultural challenges (pp. 239254). Hershey, PA: Information Science.

Edmundson, A. L. (2007). The cultural adaptation process (CAP) model. In A. Edmundson, (Ed.), Globalized e-learning cultural challenges (pp. 267-290). Hershey, PA: Information Science.

Gunawardena, C. N., Wilson, P. L., \& Nolla, A. C. (2003). Culture and online education. In M. G. Moore \& W. G. Anderson (Eds.), Handbook of distance education (pp. 753-775). Mahwah, NJ: Erlbaum.

Henderson, L. (1996). Instructional design of interactive multimedia: A cultural critique. Educational Technology Research and Development, 44(4), 85-104.

Henderson, L. (2007). Theorizing a multiple cultures instructional design model for elearning and e-teaching. In A. Edmundson, Globalized e-learning cultural challenges (pp. 130-153). Hershey, PA: Information Science.

Mason, R. (2003). Global education: Out of the ivory tower. In M. G. Moore \& W. G. Anderson (Eds.), Handbook of distance education (pp. 743-752). Mahwah, NJ: Erlbaum.

McLoughlin, C. (1999). Crossing boundaries: Curriculum and teaching implications of culturally inclusive online learning. The University of New England. Retrieved from 
http://www.aare.edu.au/o1pap/mclo1720.htm

Miles, M., \& Huberman, A. (1994). Qualitative data analysis: An expanded sourcebook (2nd ed.). Thousand Oaks, CA: Sage.

Morrison, G. R., Ross, S. M., \& Kemp, J. E. (2004). Designing effective instruction (4th ed.). Hoboken, NJ: Wiley.

Rogers, P. C., Graham, C. R. \& Mayes, C. T. (2007). Cultural competence and instructional design: Exploration research into the delivery of online instruction cross-culturally. Educational Technology, Research and Development, 55(2), 197-217.

Smith, D. R., \& Ayers, D. F. (2006). Culturally responsive pedagogy and online learning: Implications for the globalized community college. Community College Journal of Research and Practice, 30(5/6), 401-415.

Thomas, D.C. \& Inkson, K. (2009). Cultural intelligence: Living and working globally. San Francisco, CA: Berrett-Koehler.

Yin, R. K. (2003). Case study research design and methods (3rd ed.). Thousand Oaks, CA: Sage.

Athabasca University

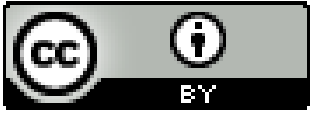

